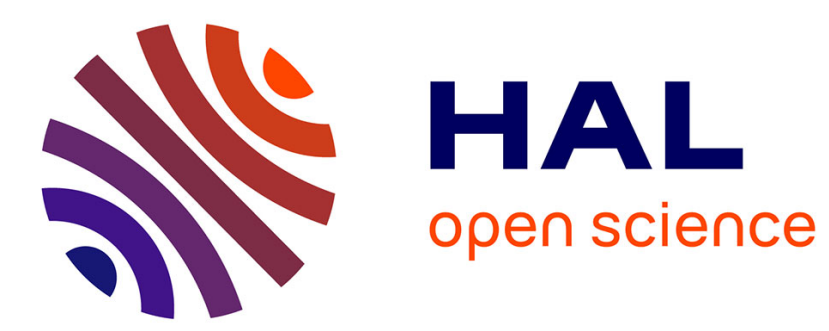

\title{
ANGULAR DISTRIBUTIONS OF PHOTOELECTRONS AND NON-THERMAL PHOTOIONS FROM ATOMS AND MOLECULES
}

J. Dehmer

\section{- To cite this version:}

J. Dehmer. ANGULAR DISTRIBUTIONS OF PHOTOELECTRONS AND NON-THERMAL PHOTOIONS FROM ATOMS AND MOLECULES. Journal de Physique Colloques, 1978, 39 (C4), pp.C442-C4-50. 10.1051/jphyscol:1978406 . jpa-00217440

\author{
HAL Id: jpa-00217440 \\ https://hal.science/jpa-00217440
}

Submitted on 1 Jan 1978

HAL is a multi-disciplinary open access archive for the deposit and dissemination of scientific research documents, whether they are published or not. The documents may come from teaching and research institutions in France or abroad, or from public or private research centers.
L'archive ouverte pluridisciplinaire HAL, est destinée au dépôt et à la diffusion de documents scientifiques de niveau recherche, publiés ou non, émanant des établissements d'enseignement et de recherche français ou étrangers, des laboratoires publics ou privés. 


\title{
ANGULAR DISTRIBUTIONS OF PHOTOELECTRONS AND NON-THERMAL PHOTOIONS FROM ATOMS AND MOLECULES $\left(^{*}\right)$
}

\author{
J. L. DEHMER
}

Argonne National Laboratory, Argonne, Illinois 60439, U.S.A.

\begin{abstract}
Résumé. - Durant ces dix dernières années, les distributions angulaires des photo-électrons ont été exclusivement utilisées pour étudier la dynamique des processus de photoionisation dans les atomes et les molécules. Nous faisons le point, ici, sur les résultats les plus marquants survenus depuis la dernière Conférence Internationale d'il y a trois ans. De loin, les plus grands progrès ont eu lieu pour les atomes, l'intérêt s'étant plus particulièrement porté sur les points suivants : amélioration de l'ordre zéro (Hartree-Slater, Cooper-Zare), compréhension du paramètre d'asymétrie $\beta(\varepsilon)$, en considérant les corrélations électroniques, les effets relativistes, les interactions de l'état final anisotropique. L'étude des gaz rares a bénéficié de l'étroite coordination entre l'expérience et la théorie, tandis que l'étude des atomes non sphériques est restée principalement théorique, mis à part les mesures récentes sur l'oxygène atomique. Les études de distribution angulaire sur les molécules sont encore à un stade très préliminaire de développement. Ceci résulte du manque de mesures en fonction de la longueur d'onde, mises à part les expériences très récentes, et l'absence de méthodes théoriques pratiques et réalistes. Le point sera donc fait sur ce travail récent, ainsi que sur une sélection de travaux plus anciens concernant les molécules et obtenus avec des raies de résonance. De plus, une nouvelle série d'études angulaires sur les molécules - la distribution angulaire des ions non thermiques formés par photoionisation dissociative - fournissant des informations complémentaires sur les mesures correspondantes avec des photoélectrons, sera présentée.
\end{abstract}

\begin{abstract}
During the last ten years, photoelectron angular distributions have been used extensively to study the dynamics of the photoionization process in atoms and molecules. Here we review some major advances in this body of work, with special emphasis on results emerging since the last Conference on VUV Radiation Physics three years ago. By far the greatest progress has occurred for atoms, where interest is focussed on improving our zero-order (Hartree-Slater, Cooper-Zare) understanding of the asymmetry parameter $\beta(\varepsilon)$, by considering electron correlations, relativistic effects, and anisotropic final-state interactions. The study of the rare gases has benefitted from extensive coordination between experiment and theory, whereas work on non-spherical atoms has been mainly theoretical, with the only measurements being performed very recently on atomic oxygen. Angular distribution studies on molecules are in a much earlier stage of development. Progress has been impeded by the lack of practical, realistic theoretical methods and wavelength-dependent measurements, both of which are becoming available only now. This recent work, together with selected topics from earlier resonance-line work on molecules will be reviewed. In addition, a new class of angle-dependent studies of molecules will be discussed - the angular distribution of non-thermal ions formed by dissociative photoionization - which provides information complementary to the related measurements on photoelectrons.
\end{abstract}

1. Introduction. - Photoelectron angular distributions are an important probe of photoionization dynamics because they depend on both the magnitudes and relative phases of the dipole amplitudes (dipole matrix elements) of alternative photoionization channels. The dependence on relative phases results from interference between degenerate photoionization channels and provides the opportunity to achieve a more detailed understanding of photoionization

(*) Work performed under the auspices of the U.S. ERDA. than that afforded by the study of integrated crosssections which depend only on the magnitudes of the dipole amplitudes. At the same time, the more complicated nature of photoelectron angular distributions puts a greater premium on strong interaction between theory and experiment which, in turn, requires wavelength-dependent measurements to be most effective. In what follows, we review several diverse examples of angular distribution studies and place special emphasis on cases in which theory and experiment have been combined to reveal something 
new about the photoionization process. We will also discuss a new class of experiment [1] photoion angular distributions from dissociative photoionization. These measurements yield branching ratios for degenerate photoionization channels which can be used to unravel the information contained implicitly in the photoelectron asymmetry parameter. In favorable cases, it is possible to determine the magnitudes and relative phases of the dipole amplitudes for degenerate ionization channels separately, thus completely specifying the dynamics of the photoionization process.

Strictly speaking, efforts to map out the spatial distributions of photoelectrons began in the early 1920 's with experiments employing $X$ rays $[2,3]$. Nevertheless, substantial progress began only. forty years later when both experimental and theoretical tools were available for application to the VUV photoionization of gaseous targets. The modern wave of experimental work began [4-9] in the mid1960 's shortly after the development of UV photoelectron spectroscopy. Much of the early work was plagued by large errors, usually underestimating the anisotropy of photoelectron angular distributions; but improved experimental technique and the awakening of theoretical interest quickly led to more accurate numbers $[6,8,9]$. By the early 1970's work on the rare gases had converged to the presently accepted values [10-22] and interest became focussed on detailed comparison between wavelength-dependent measurements and theoretical predictions based on Hartree-Slater [23, 24], Harîree-Fock [24], and RPAE [25] calculations which had become available. This represents the point of departure for this paper.

From the theoretical point of view, the essentials were known by the early 1930's and were summarized in Bethe's article [26] in Handbuch der Physik. For instance, early work by Sommerfeld's group [27] and some unpublished work by Oppenheimer [28] established the basic form $\mathrm{d} \sigma / \mathrm{d} \Omega=a+b \cos ^{2} \theta$, which applies to a randomly oriented target in the dipole approximation. However, the generality of the result was not emphasized and the treatment of the interference term was oversimplified, e.g., only the Coulomb part of the phase shift difference was considered. Subsequently, Yang [29] proved that photoelectron angular distributions for any randomly oriented target in the dipole approximation has this general form regardless of the internal structure of the target. This general result is now commonly written in the form

$$
\mathrm{d} \sigma / \mathrm{d} \Omega=\sigma / 4 \pi\left[1+\beta P_{2}(\cos \theta)\right],
$$

where $\sigma$ is the integrated cross-section,

$$
P_{2}(\cos \theta)=\frac{1}{2}\left(3 \cos ^{2} \theta-1\right),
$$

and $\theta$ is the photoelectron's direction relative to the electric vector of linearly polarized light, in order to focus the study of photoelectron angular distributions on a single parameter, $\beta$, the so-called asymmetry parameter. Theoretical expressions for $\beta$ for both atoms [30-36] and molecules [31, 32, 37-41] began appearing just as modern measurements using VUV and soft X-ray light sources were producing accurate results. The work of Cooper and Zare [30] which expressed $\beta$ for atoms in an independent electron, LS-coupled scheme has had a great impact on the work described below, because it represents an easily applicable and realistic level of sophistication and because it emerged at a time when practical methods to compute one-electron photoionization cross-sections for atoms had just begun to be exploited extensively (see e.g. ref. [42]). Therefore although much has been learned and predicted regarding non-Cooper-Zare effects, the model constitutes a very realistic representation for a large range of cases and a useful frame of reference for showcasing, e.g., anisotropic (final-state) interactions. The early work in the molecular field had no such vehicle for early exploitation and progress on molecular asymmetry parameters has been more spotty.

A recent highlight in the development of angular distribution theory is the introduction and subsequent application of the $j_{\mathrm{t}}$ representation by Dill and co-workers $[31,32,34,36,39,43]$. The crux is the indexing of ionization channels, not by their total angular momentum, $J$, but by the amount of angular momentum transferred in the photoionization process

$$
\mathbf{j}_{\mathrm{t}} \equiv \mathbf{J}_{\mathbf{c}}+\mathbf{s}-\mathbf{J}_{\mathrm{o}}=\mathbf{j}_{\gamma}-\mathbf{I}
$$

where the subscripts $c, o$, and $\gamma$ refer to the ion core, the initial state, and the photon, respectively, and $(l, s)$ are the orbital and spin angular momenta of the ejected electron. Under these circumstances, the differential cross-section factors into separate contributions for the alternative values $j_{\mathfrak{t}}$ of the angular momentum transfer. The motivation for this choice of basis is twofold : First, transition amplitudes for ionization with alternative values of $j_{\mathrm{t}}$ superimpose incoherently to form the differential cross-section. (Such a factorization is not possible in terms of the total angular momentum $J$, since dipole amplitudes with alternative values of $J$ superimpose coherently.) Second, each $j_{1}$ contributes a characteristic angular dependence to the differential cross-section. This characteristic geometry is determined according to the parity change $\pi_{0} \pi_{\mathrm{e}}$ and relates to classes of interactions experienced by the departing photoelectron. This point of view has been beneficially employed in cases to be discussed below. Note that the CooperZare formula is obtained from this formulation in the limit of LS coupling and negligible dynamical coupling of the photoelectron's orbital momentum to the net orbital motion of the residual ion, leading to a single value of $j_{\mathfrak{t}}=l_{0}$. 
2. Atoms. - 2.1 Electron CORRelation efFects IN RARE GASES. - Photoelectron angular distribution studies on the rare gases are a prime example of how a fruitful interaction between experiment and theory can improve our understanding of photoionization dynamics. Here the central problem is the elucidation of alternative electron correlations which play an important role in photoionization of a sequence of atoms with increasingly complex shell structure. The information available in this case is excellent : First, there are accurate, wavelengthdependent measurements with good agreement between several laboratories [8-22]. Second, corresponding calculations exist at the HartreeSlater [23, 24], Hartree-Fock [24], and RPAE [25] levels. These calculations are all based on the CooperZare formulation which was both readily available when these calculations began, and has since been shown [36] to be a good approximation for closedshell atoms.

The information resulting from this well-balanced set of studies emerges qualitatively in figure 1 where the experimental and theoretical values of the asymmetry parameter are given for the outer $p$ shell of $\mathrm{Ne}, \mathrm{Ar}$, and $\mathrm{Xe}$. The $\mathrm{Ne} 2 \mathrm{p}$ subshell is the point of departure for this comparison since there is excellent agreement between experiment $[14,19,21]$ and HS (not shown), HF [24], and RPAE [25] calculations. As will become apparent below, this follows from the absence of a $d$ subshell in the shell of the initial $2 \mathrm{p}$ state. In heavier rare gases, strong intra-shell correlations involving the energetically and spatially nearby d-type states induce large differences in the alternative calculations which treat these correlations differently. This type of correlation, however, is vanishingly small for nodeless initial states such as the $2 p$ subshell of Ne. For the Ar 3p, interactions between the $3 p$ subshell and d-type states become strong with the results in figure 1 : Now the HF [24] deviates significantly from the HS (not shown) because the latter neglects the strong exchange interaction between the photoelectron (predominantly $l=2)$ and the 3 p hole. Further, the HF and RPAE [25] exhibit significant deviations because the HF neglects the important electron correlation effect of configuration interaction of the ground $\left(3 p^{6}\right)$ configuration of argon with states of the type $\left(3 \mathrm{p}^{4}, \mathrm{nd}^{2}\right)$ which are included in RPAE, MBPT, and $R$-matrix methods. The good agreement between experiment $[16,19]$ and RPAE tells us clearly that the essential interactions have been successfully accounted for at this level of approximation. As is well known, the dip in $\beta$ between $30-40 \mathrm{eV}$ is indicative of the Cooper minimum in the $3 p \rightarrow \varepsilon d$ channel. Clearly, $\beta$ is quite sensitive to the location of the minimum since, when the $3 \mathrm{p} \rightarrow \varepsilon \mathrm{d}$ matrix element vanishes, only the $3 p \rightarrow \varepsilon s$ occurs and the photoelectron angular distribution is entirely an $\mathrm{s}$ wave which is isotropic. Thus, $\beta$ must be zero at this point, as seen in figure 1 .
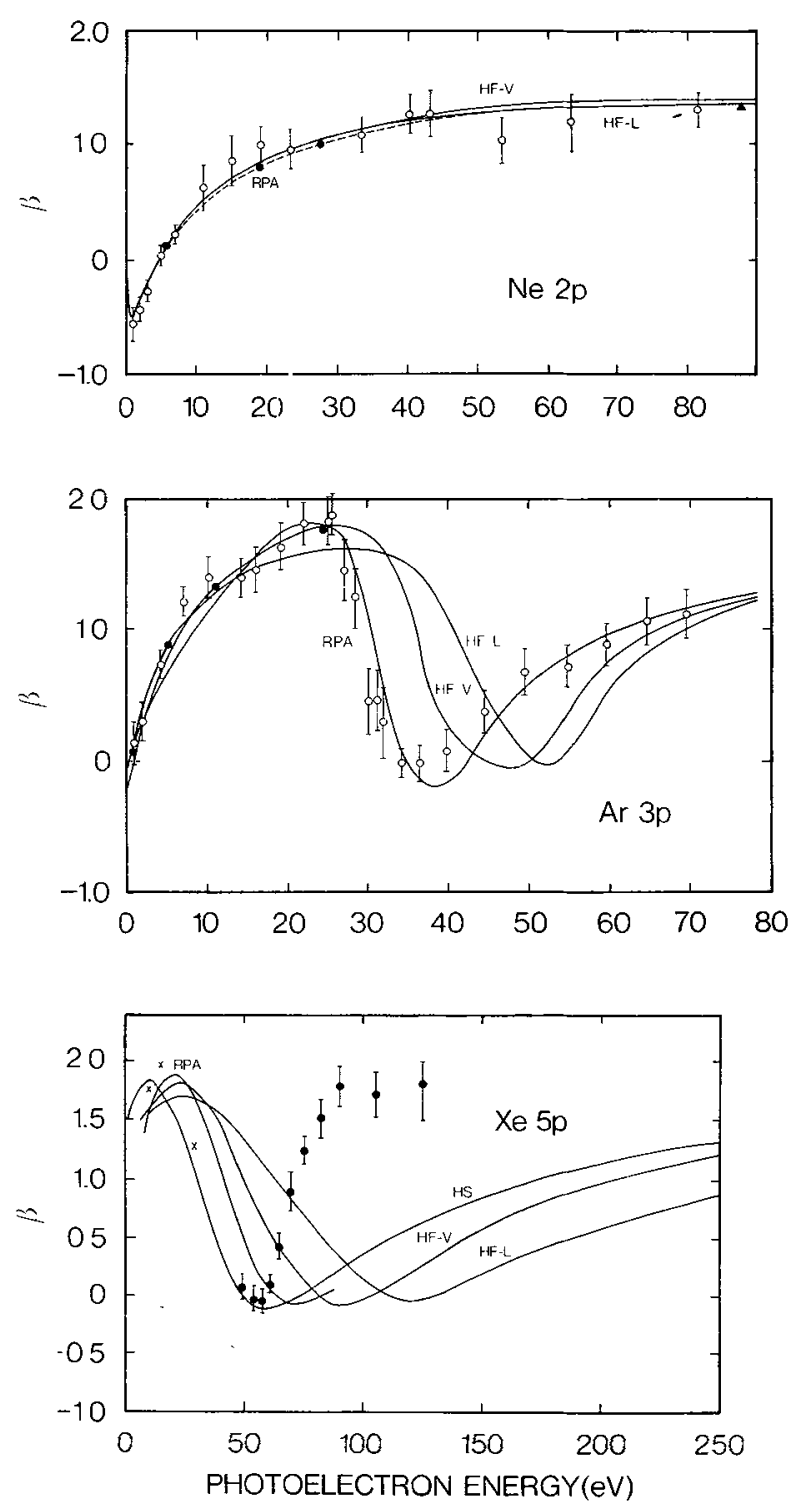

Fig. 1. - Asymmetry parameters for the Ne 2p, Ar 3p, and Xe $5 p$ subshells. For Ne 2p, RPA-Ref. [25], HF-L and HF-V-Ref. [24], $\bullet-R e f$. [19], O-Ref. [21], $\Delta$-Ref. [14]. For Ar 3p, RPA-Ref [25]. HF-L and HF-V-Ref. [24]. -Ref. [19]. O-Ref. [16] For Xe 5p, RPA-Ref. [25], HF-L, HF-V, and HS-Ref. [24], $x-\operatorname{Ref}$ [19], - - Ref. [20].

Since excitation in $\mathrm{Ne}$ is from the $2 \mathrm{p}$, no Cooper zero is possible, and the large oscillation in $\beta$ is absent.

The asymmetry parameter for the xenon $5 \mathrm{p}$ photoelectron angular distribution is also shown in figure 1. Here we find only fair agreement between experiment $[19,20]$ and RPAE theory [26] at low photoelectron energy and very poor agreement at higher energies. There are two qualitative differences between argon $3 \mathrm{p}$ and xenon $5 \mathrm{p}$ which might relate to the difference in agreement with experiment in the two cases. First, xenon is heavy enough so that relativistic effects (particularly spin-orbit coupling) are becoming important, but this is not thought to be crucial on the energy scale considered here. Second, xenon 
has an inner $4 \mathrm{~d}$ subshell, while ground state argon has no $\mathrm{d}$ electrons, and this is thought to be the reason for the differences in the two cases : An important correlation for xenon $5 p$ is the interchannel interaction between $5 \mathrm{p} \rightarrow \varepsilon \mathrm{d}$ and the very strong $4 \mathrm{~d} \rightarrow \varepsilon f$ channel. The $4 \mathrm{~d}$ channel opens up about $55 \mathrm{eV}$ above the $5 \mathrm{p}$, and from figure 1, it is seen that this is just where the discrepancy gets large. The RPAE calculation did not include this interchannelcoupling type of electron correlation, and its neglect is seen to have dramatic effects. Therefore, comparison of accurate, wavelength-dependent measurements with calculations which include alternative types of electron correlations clearly broadcasts which of these interactions is essential to accurate representations of the increasingly complex atoms.

2.2 ANISOTROPIC FINAL-STATE INTERACTIONS IN OPEN-SHELL ATOMS. - In general, it will be necessary to go beyond the Cooper-Zare independent-particle formulation of $\beta$ in order to treat anisotropic interactions between the outgoing electrons and the residual ion. This is most easily visualized in terms of a twostep process. In the first stage, the photon is absorbed by the target, imparting an angular momentum $j_{y}=1$ to the photoelectron. During this step, the angular momentum transferred to the target ion takes on the single value $j_{\mathbf{t}}=l_{0}$ only. In CooperZare level formulations, the photoelectron then

escapes to infinity with no further exchange of angular momentum. In general, and for open-shell atoms in particular, a second stage must be considered in which the photoelectron and the core undergo anisotropic interactions during the photoelectron's escape, which can lead to further exchange of angular momentum. In this case, $j_{\mathbf{t}}$ is free to take on values other than $l_{0}$, which can lead to additional terms in the expression for $\beta(\varepsilon)$. Consideration of this very important aspect of the photoionization process is at the heart of the so-called angular-momentumtransfer formulation by Dill and co-workers [31, 32, $34,36,39,43]$. Other formulations of equivalent detail exist (e.g., ref. [33]) ; however, the $j_{\mathrm{t}}$ representation has the distinct advantage of displaying explicitly the alternative contributions arising in the different stages of the photoionization process.

Since, for open-shell atoms, the photoelectron-ion interaction is always anisotropic, depending on both the multiplet term of the residual ion and the total orbital momentum of the ion-photoelectron finalstate channel, practically all development in the subfield has concentrated on open-shell systems. Calculations have been carried out for $\mathrm{O}(2 \mathrm{p})[44,45]$, $\mathrm{S}(3 \mathrm{p})[36,46], \mathrm{Cl}(3 \mathrm{~s})$ [47], Cl(3p) [48], Se(4p) [49], $\mathrm{Br}(4 \mathrm{p})$ [49], $\mathrm{Te}(5 \mathrm{p})$ [50] and $\mathrm{I}(5 \mathrm{p})$ [50] in LS coupling using Hartree-Fock wavefunctions. The results can be summarized as follows : First, the phase-shift differences among the dominant $l \rightarrow l+1$ channels associated with alternative terms of the residual ion constitute a useful barometer for the degree of anisotropic interactions. In practice, phase-shift differences must be in excess of 0.1 rd to produce measurable effects in $\beta$ for the various channels. Second, first-row atoms $[44,45]$, such as atomic oxygen, fail this test so that a plot of $\beta$ for the ${ }^{4} \mathrm{~S},{ }^{2} \mathrm{P}$, and ${ }^{2} \mathrm{D}$ terms of $\mathrm{O}^{+}$, on a common kinetic energy scale, would be nearly identical. Third, second-row atoms, such as $\mathrm{S}$ and $\mathrm{Cl}$, exhibit easily measurable effects. These effects then decrease slowly upon going to the third and fourth rows, indicating that anisotropic interactions maximize at $n=3$. Fourth, anisotropic effects can be observed in photoionization of an $s$ subshell of an open-shell atom. Starace et al. [47] have demonstrated dramatic deviations from the rigorous independent-particle result $\beta=2$ for 3s photoionization in atomic chlorine. (Analogous effects $[10,51]$ in closed-shell atoms are discussed below in the context of relativistic effects.) The main shortcoming in this subfield is the difficulty of making measurements on the systems of greatest interest. Only very recently, measurements have been performed on atomic oxygen $[52,53]$, but as noted above, anisotropic interactions are not strong in first-row atoms. Perhaps this problem will be best resolved by measurements on molecules (see section 3), whose inherently anisotropic field will ensure strong anisotropic effects in photoelectron angular distributions [40].

\subsection{RELATIVISTIC AND NON-DIPOLE EFFECTS. -} A third major subfield.in the study of photoelectron angular distributions involves relativistic and nondipole effects. An initial semi-quantitative mapping of the effects of spin-orbit coupling was conducted by Walker ef al. $[35,54,55]$ in the dipole approximation utilizing the Dirac-Slater model. This work was a useful qualitative illustration of some of the consequences of a small displacement in energy and radial distribution between the spin-orbit sublevels of a non-s initial and/or final-state wavefunction. For example, deviations from $\beta=2$ for s-type initial states were demonstrated $[35,55]$. This deviation is particularly strong for alkali atoms [35] in the vicinity of the Cooper zero, where $\beta$ oscillates over two cycles and reaches minimum values near $\beta \sim 0$. As recognized by Walker and Waber [35], these conclusions must be reconsidered in light of higher order interactions with the radiation field, especially in the case where the dipole-matrix elements are near zero. Although the effects of spin-orbit coupling are largest near a Cooper zero, they also occur for other systems such as the Xe 5s orbital, which was predicted [55] to have a $\beta$ beginning at $\beta=-1$ at threshold and approaching its non-relativistic value of $\beta=2$ near 20-30 eV kinetic energy. This has been tested experimentally by Dehmer and Dill [51] who reported a value of $\beta=1.4 \pm 0.1$ at $304 \AA$, to be compared with the calculated value [55] of $\beta=1.7$. Dehmer 
and Dill [51] also wrote out a general expression for $\beta$ in the $j_{\mathbf{t}}$ representation, and showed the hierarchy of approximations leading to the simplified form for $\beta$ used by Walker and Waber [55]. Further computational work is necessary to resolve, from among the several possible causes, the remaining deviation between the single measurement and the DiracSlater calculation. An analogous measurement on the $\mathrm{Hg} 6 \mathrm{~s}$ by Niehaus and Ruf [10] offers the same evidence on another system, where again a DiracSlater calculation [56] underestimates the deviation from $\beta=2$. Another example of spin-orbit effect concerns the angular distributions of the $5 \mathrm{p}_{1 / 2,3 / 2}$ subshells of $\mathrm{Xe}$ and the $5 \mathrm{~d}_{3 / 2,5 / 2}$ subshells of $\mathrm{Hg}$. The Dirac-Slater calculations [55] show small but measurable differences between the $\beta$ curves for the spin-orbit sublevels; however, synchrotron measurements have heretofore not reported separate $\beta$ 's for spin-orbit sublevels, and the resonance line work, which does resolve the substates, is too sparse to clearly substantiate the predictions. In this case, the gross, qualitative aspects of the calculations are probably valid, but the details should not be taken too seriously until some accurate calculations, are completed.

Very recently this type of study has been taken one step further (inclusion of both relativistic and non-dipole effects). Again using the Dirac-Slater model, Pratt and coworkers [56, 57] have demonstrated significant deviations from the non-relativistic dipole approximation for both low- and high- $Z$ atoms. In light elements these effects are found in the $\mathrm{keV}$ range (and even below). In heavy elements, they are found even for energies very close to threshold. For total cross-sections, by contrast, the surviving integrated relativistic and multipole corrections tend to cancel, so that the non-relativistic dipole approximation holds to surprisingly high energies. This work contains those effects discussed above (e.g., the $5 \mathrm{~s}$ of $\mathrm{Xe}$ ) but in addition, maps out multipole effects manifested in the more general formulation for photoelectron angular distributions,

$$
\frac{\mathrm{d} \sigma}{\mathrm{d} \Omega}=\frac{\sigma}{4 \pi} \sum_{n=0}^{\infty} B_{n} P_{n}(\cos \theta) .
$$

In this expansion, we can identify the dipole asymmetry parameter $\beta=-2 B_{2}$; in addition, however, there are non-dipole terms which are being shown to be significant in many circumstances for both light and heavy atoms. An obvious consequence of significant non-dipole effects is that branching ratio measurements made at the so-called magic angle [58] $\theta=54^{\circ} 44^{\prime}$ would not be rigorously independent of differences in photoelectron angular distributions, e.g., only the $P_{2}(\cos \theta)$ term vanishes in the above expression, leaving angle-dependent contributions from $B_{n}$ with $n>2$. Examples of non-dipole effects can be found in experiments on Ne 2p subshells [14] and the $\mathrm{Kr} 3 \mathrm{~d}$ subshell [6]. Further details of extensive evaluations of the coefficients $B_{n}$ are being prepared for publication [57].

2.4 INDIRECT PROCESSES. - The determination of the spectral variation of photoelectron angular distributions through autoionizing resonances is an essentially untapped resource of dynamical information on photoionization. Theoretical analyses $[34,59,60]$ on the autoionizing Rydberg series leading to the ${ }^{2} \mathrm{P}_{1 / 2}$ thresholds in $\mathrm{Xe}$ and $\mathrm{Ar}$ demonstrated not only sharp spectral variations of the angular distributions across resonance features, but more importantly, angular distributions that should depart markedly from those predicted by direct (nonresonant) ionization models, such as the CooperZare model. Deviations from direct ionization predictions arise owing to the enhancement by the autoionizing process of the effects of just those forces that are often sufficiently weak as to go undetected in non-resonant photoionization. In the case of $\mathrm{Xe}$, the gross trends predicted by Dill [34] were later confirmed by Samson and Gardner [61] in the vicinity of the 7,8d and 8,9s autoionizing Rydberg lines leading to the ${ }^{2} \mathrm{P}_{1 / 2}$ threshold of Xe. To date, other experimental studies of the variation of $\beta$ through autoionizing lines have not been performed; however, the use of synchrotron radiation should make this possible in the near future.

3. Molecules. - 3.1 Photoelectron angular DisTRIBUtions. EXPERIMENTS. - Nearly all angular distribution measurements on molecules have thus far been limited to $\mathrm{He} \mathrm{I}$ and $\mathrm{Ne} I$ line sources. As stressed above, this is a severe limitation which must be overcome before angular distributions can be fully used to study photoionization dynamics. This is widely recognized and work on this problem has recently begun at Daresbury, but as of this writing, the preliminary results are not generally available.

In spite of the limited wavelengths used, however, angular distribution studies have been used to very good advantage in molecules to study aspects of the photoionization process related to the internal rovibronic structure. In a sense, such internal structure substitutes for variable wavelength light since, e.g., population of alternative levels of a vibrational progression leads to photoelectrons with varying amounts of kinetic energy. We illustrate the utility of resonance line work in the case of molecules with a few examples : First, angular distribution measurements can be used as a qualitative spectroscopic tool to obtain partial resolution of overlapping electronic bands in photoelectron spectra. Carlson et al. [62] demonstrated this use clearly in the case of benzene, whose complicated photoelectron spectrum contains several overlapping diffuse electronic states. By measuring $\beta$ at closely spaced intervals, the presence of the contributing electronic states 
was clearly indicated by plateaus. Second, measurement of $\beta$ as a function of vibrational level within an electronic band can yield information on the $R$ dependence and/or kinetic energy dependence of dipole amplitudes, as well as on alternative ionization mechanisms. For example, photoionization of $\mathrm{O}_{2}$ with the $16.85 \mathrm{eV} \mathrm{Ne} \mathrm{I}$ resonance line produces the ground state of $\mathrm{O}_{2}^{+}$in over 14 vibrational levels [63]. The $\beta$ for the first 4 is $\sim-0.6$, whereas the $\beta$ for the upper vibrational levels is $\sim 0$. This implies the existence of different mechanisms for producing these alternative sets of final states. This situation is clarified by two facts : At $584 \AA$ only the first four or five vibrational levels are populated by direct photoionization, whereas $\lambda=736 \AA$ hits an autoionizing line in the photoabsorption spectrum [64] of $\mathrm{O}_{2}$. This suggests that the lower levels are produced by direct photoionization, and the upper levels by the indirect process. Strong $v$-dependence of $\beta$ is also observed [63] in production of the ground state of $\mathrm{N}_{2}^{+}$by photoionization of $\mathrm{N}_{2}$ at $584 \AA$. This has been tentatively attributed [65] to a strong $R$-dependence in the dipole amplitudes and channel phase shifts. The final example involves dependence of the angular distribution on rotational quantum number. Measurements of $\beta$ for partially resolved rotational levels of $\mathrm{H}_{2}$ by Niehaus and Ruf [66] indicate that the photoelectron angular distribution is a strong function of the change in rotational quantum number, $\Delta N$. This was interpreted by Dill [39], who showed how transitions with alternative $\Delta N$ correspond to alternative possible $j_{\mathrm{t}}$ 's and therefore lead automatically to different values of the asymmetry parameter. The agreement between experiment and theory has recently been improved [67] by consideration of dynamic coupling between the photoelectron and the rotational motion of the ion core.

\subsection{Photoelectron angular Distributions.} THEORY. - Theoretical progress on angular distributions of molecules has likewise been spotty, and, as of yet, no flexible, realistic means is readily available for the purpose of mapping the wavelengthdependent $\beta$ 's for molecules of arbitrary complexity, although this problem may be overcome soon. A large volume of earlier work (see, e.g., [68]) in this area utilized plane wave or orthogonalized plane waves for final states. Owing to the importance of phase shift information to angular distributions, these models, which neglect both the Coulomb field of the ion and the phase shift of photoelectrons, cannot be considered realistic in the VUV ênergy range. Use of orthogonalized Coulomb waves [69] employs a better point of departure and exhibits some gross agreement with experiment ; but this, too, suffers from the complete lack of phase shift information and is not discussed further here. Contributions to this field which employ methods containing all the essential information (e.g., molecular symmetry, proper asymp- totic boundary conditions, phase shifts, orthogonality to core levels, etc.) include the work on $\mathrm{H}_{2}$ [37] and $\mathrm{N}_{2}$ [65] using a single-center-expansion representation, Dill's analysis [39] of the effect of rotational autoionization in $\mathrm{H}_{2}$ using multichannel quantum defect theory, recent work [40,70-72] on $\mathrm{N}_{2}$ and $\mathrm{CO}$ using the multiple-scattering model [73], and studies [74,75] of the dependence of $\beta$ on rotational and vibrational states in $\mathrm{H}_{2}$ using an ellipsoidal coordinate system. The multiple-scattering work has recently been generalized to molecules of arbitrary symmetry and will be useful for extensive and realistic mapping of asymmetry parameters for molecules of arbitrary complexity and over broad energy ranges. We also anticipate, in the relatively near future, the development of an $R$-matrix method for treatment of these problems.

In order to illustrate the use of theory to study molecular angular distributions, we discuss briefly $\mathrm{K}$-shell photoionization in $\mathrm{N}_{2}$ and $\mathrm{CO}$. Total and differential photoionization cross sections for these processes, calculated recently using the multiplescattering model [40,70,71], exhibit a very prominent shape resonance in the $\sigma_{\mathbf{u}}$ ionization channel at $\sim 0.8 \mathrm{Ry}$ above the respective $\mathrm{K}$-shell thresholds. As described in detail elsewhere $[40,70]$, this resonance, also observed experimentally [76], is due to the $l=3$ component of the $\sigma_{\mathrm{u}}$ molecular continuum wavefunction, which indicates two crucial aspects of molecular photoionization : First, although K-shell photoionization produces a pure $\mathrm{p}$ wave in an atomic core, the anisotropic molecular field can scatter this outgoing $\mathrm{p}$ wave into a whole range of $l$ values outside the molecule. (This prompted the earlier statement in section 2.2 that molecules are the best testing ground for anisotropic effects.) Second, high-l contributions are very common-place in molecular continuum dynamics due to the spatial extent of the molecular field. That is, an f-wave resonance is prominent in photoionization of $\mathrm{N}_{2}$, while this is not the case for the separated atom, N, or the united atom, Si. The asymmetry parameters for K-shell photoionization in $\mathrm{N}_{2}$ and $\mathrm{CO}$ [70] show two dramatic molecular effects. First, they all deviate significantly from $\beta=2$ (the rigorous value for $\mathrm{K}$-shell photoionization in the absence of anisotropic interactions on the photoelectron) for more than $100 \mathrm{eV}$ above the respective thresholds. Second, each reflects the shape resonance discussed above, although the effects are much larger for $\mathrm{CO}$ than for $\mathrm{N}_{2}$, for reasons discussed fully in reference [70]. We note also that the fixed-molecule photoelectron angular distributions for K-shell photoionization of $\mathrm{N}_{2}$ and $\mathrm{CO}$ have also been calculated [71], demonstrating graphically that the photocurrent at the shape resonance has an $\mathrm{f}$-wave distribution in space. A similar calculation for valence shells of $\mathrm{N}_{2}$ and $\mathrm{CO}$ has been performed by Davenport [72] with the same major conclusions. (An important application of these reso- 
nance effects in the context of oriented-molecule photoelectron angular distributions is the determination of the orientation of adsorbed molecules; however, this field is outside the scope of this review, and will not be discussed here. For a general overview, see ref. [77]). Therefore, multiple-scattering techniques have proven very powerful in interpreting major, independent-electron photoionization features of diatomics and are presently being adapted to the problem of performing survey calculations in molecules of arbitrary symmetry. This provides the promise of closer interaction between experiment and theory in the all-important case of molecules, while we await the development of more sophisticated theoretical methods.

3.3 Photoion angular Distributions. - The measurement of angular distributions and kinetic energies of photodissociation products yields, respectively, information on the symmetry and internal energy of the dissociating parent state(s). This can be a uniquely powerful spectroscopic tool, particularly in regions of the photoabsorption spectrum dominated by diffuse bands which are characteristic of repulsive final states. Moreover, such measurements can provide dynamical information on the photoexcitation process, such as branching ratios and dissociation lifetimes. There is significant literature on the theoretical (see e.g., refs. [78-81]) and experimental (see, e.g., refs. [78, 81-83]) aspects of this facet of molecular photodissociation; however, it is generally confined to the spectral range below the ionizing potential.

In dissociative photoionization, a new wrinkle emerges : When a molecular ion is produced in a repulsive state by photoionization, the process also involves the ejection of photoelectrons, which, in many cases, have available to them degenerate ionization channels of more than one symmetry type. Since the photoion angular distribution yields symmetry information about the total final state (molecular ion plus photoelectron), this complicates the determination of the symmetry of the repulsive ionic state alone. On the other hand, when the repulsive state is known, this affords us the rare opportunity of directly measuring the branching ratio for the alternative, degenerate ionization channels. This branching ratio cannot be obtained from the electron energy spectrum owing to degeneracy; and the photoelectron angular distribution yields an implicit function of both the branching ratio and the relative phases of the alternative ionization channels from which the branching ratio cannot be isolated without further information. Once the branching ratio information is obtained from the photoion angular distribution, however, it can be used to help unravel the information contained in the photoelectron asymmetry parameter. Under favorable conditions, the combination of photoion and photoelectron angular distributions can lead to a full determination of the dipole amplitudes and relative phases of the ionization channels, thus completely specifying all of the dynamical parameters of the photoionization process.

To illustrate the application of this new type of angular distribution measurement, we review a recent measurement [1] of photoion angular distributions from dissociative photoionization of $\mathrm{H}_{2}$ at $304 \AA$. The potential energy curves [84] for states of $\mathrm{H}_{2}$ and $\mathrm{H}_{2}^{+}$ important in our discussion of dissociative photoionization of $\mathrm{H}_{2}$ at $\lambda=304 \AA$ are given in figure 2 . The shaded area denotes the Franck-Condon region of the $v=0$ vibrational level of the ground state of

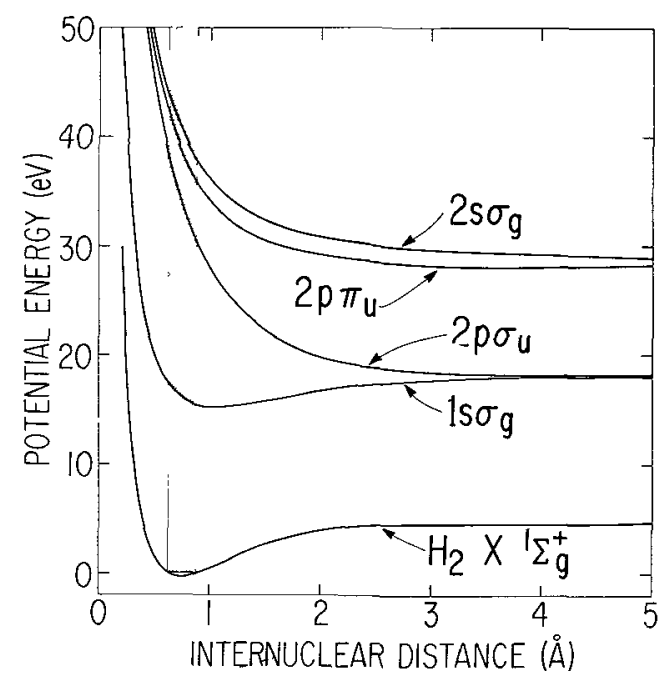

Fig. 2 - Selected potential energy curves (taken from Ref. [84]) for states of $\mathrm{H}_{2}$ and $\mathrm{H}_{2}^{+}$which participate in dissociative photo1onization at $\lambda=304 \AA$. The shaded region represents the FranckCondon region associated with the $\mathrm{H}_{2} \times{ }^{1} \Sigma_{\mathrm{g}}^{+}(v=0)$ ground state.

$\mathrm{H}_{2}$. Only transitions to those portions of the finalstate potential-energy curves lying within this region will be appreciably populated in the photoionization process, with the excess energy being carried off by the photoelectron. Although $\sim 2 \%$ of the transitions to the ground $1 \mathrm{~s} \sigma_{\mathrm{g}}$ ionic state of $\mathrm{H}_{2}^{+}$are dissociative [85], the photofragment energy is very low and is not separated from the very intense $\mathrm{H}_{2}^{+}$peak in this work. The more energetic protons, which we are primarily concerned with, result from the dissociation of the three repulsive curves of $\mathrm{H}_{2}^{+}$lying above the $1 \mathrm{~s} \sigma_{\mathrm{g}}$ ground state. These states of the ion have $2 \mathrm{p} \sigma_{\mathrm{u}}, 2 \mathrm{p} \pi_{\mathrm{u}}$, and $2 \mathrm{~s} \sigma_{\mathrm{g}}$ symmetries are all the product of a two-electron excitation process.

Figure 3 contains representative photoion energy spectra. Samson and Gardner [86] assigned the $5.5 \mathrm{eV}$ band to formation of the $2 \mathrm{p} \pi_{\mathrm{u}}+2 \mathrm{~s} \sigma_{\mathrm{g}}$ states. There is evidence $[86,87]$ that formation of the $2 \mathrm{p} \pi_{\mathrm{u}}$ dominates at this wavelength. The $8 \mathrm{eV}$ band is known, on energetic grounds, to correspond to protons from the dissociation of the $2 \mathrm{p} \sigma_{\mathrm{u}}$ state. The two spectra in figure 3 permit qualitative observation that the $5.5 \mathrm{eV}$ band is peaked forward, 


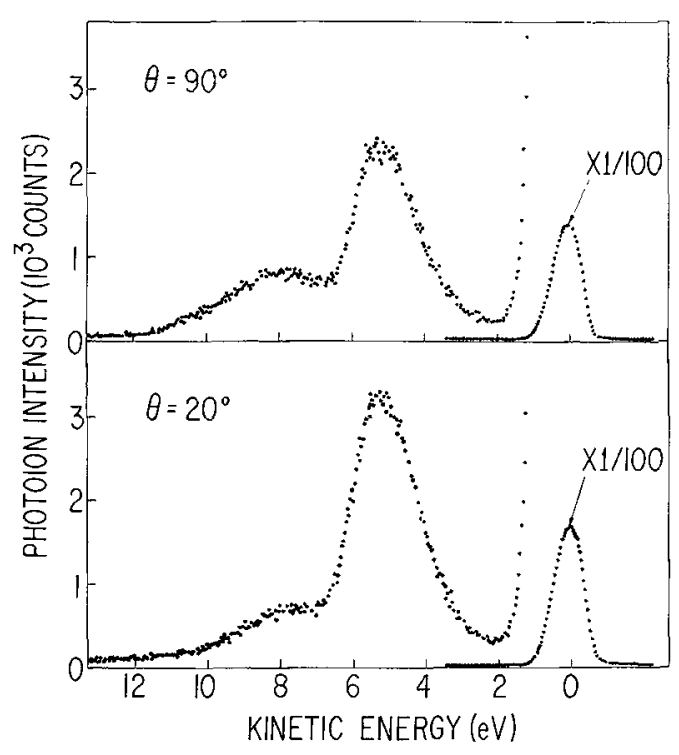

FIG 3. - Photolon kinetic energy spectra for photoonization of $\mathrm{H}_{2}(\lambda=304 \AA)$ at two observation angles $\left(\theta=20^{\circ}, 90^{\circ}\right)$ relative to incident unpolarized light beam. The large peak near zero kinetic energy is comprised mostly of $\mathrm{H}_{2}^{+} 1 \mathrm{~s} \sigma_{\mathrm{g}}$ ground state tons which have a thermal energy distribution.

along the direction of the light, while the $8 \mathrm{eV}$ band is slightly larger perpendicular to the light direction. In the detailed analysis given elsewhere [1], the average $\beta_{\mathrm{H}^{+}}$values for the $5.5 \mathrm{eV}$ and $8 \mathrm{eV}$ bands are $\sim-0.3$ and +0.9 , respectively. In a theoretical analysis described elsewhere [1], it is shown that in the axial-recoil approximation (fragment kinetic energy $\gg$ rotational spacing), the $\beta$ value for pure
$\Sigma \rightarrow \Sigma$ transitions is +2 , while that for pure $\Sigma \rightarrow \Pi$ transitions is -1 , where the capital Greek letters refer to the total symmetry of the system. Hence, the ratio of cross sections for production of final states of total symmetry $\Sigma$ and $\Pi$ is given by [1]

$$
\frac{\sigma(\Sigma \rightarrow \Sigma)}{\sigma(\Sigma \rightarrow \Pi)}=\frac{1+\beta_{\mathrm{H}^{+}}}{4-2 \beta_{\mathbf{H}^{+}}} .
$$

In the case of production of the $2 \mathrm{p} \sigma_{\mathrm{u}}$ dissociative state of $\mathrm{H}_{2}^{+}$

$$
\frac{\sigma(\Sigma \rightarrow \Sigma)}{\sigma(\Sigma \rightarrow \Pi)}=0.82
$$

where $\Sigma \rightarrow \Sigma$ and $\Sigma \rightarrow \Pi$ transitions lead to the production of $\mathrm{H}_{2}^{+}\left(2 \mathrm{p} \sigma_{\mathrm{u}}\right)$ plus a photoelectron with symmetries $\sigma_{\mathrm{g}}$ and $\pi_{\mathrm{g}}$, respectively. The interesting aspect of this result is that production of $\sigma_{\mathrm{g}}$ and $\pi_{\mathrm{g}}$ photoelectrons is roughly equal. Since the $\pi_{\mathrm{g}}$ wavefunction has a lead term $l=2$, this proves that $\mathrm{d}$ waves contribute significantly to photoionization of $\mathrm{H}_{2}$ at $304 \AA$. Other ramifications of these measurements are described in the full report [1]. Generally, a high level of detail results which is ideal for comparison with theory, in addition to the insight of the type provided by the above example.

Acknowledgments. - I wish to thank Drs. Dan Dill, Steve Manson, Mitio Inokuti, and J.-P. Desclaux for discussions, materials, and assistance in preparing this article.

\section{References}

[1] Dehmer, J. L. and Dan Dill, Phys. Rev. A, to be published.

[2] Bothe, W., Z. Phys. 26 (1924) 59

[3] Loughridge, D. H., Phys. Rev. 26 (1925) 697.

[4] Berkowitz, J. and Ehrhardt, H., Phys. Lett. 21 (1966) 531 ; and

Berkowitz, J., Ehrhardt, H. and Tekati, T., Z. Phys. $\mathbf{2 0 0}$ (1967) 69

[5] McGowan, J. W., Vroom, D. A. and Comeoux, A. R., $J$. Chem. Phys. 51 (1969) 5626.

[6] Krause, M. O., Phys. Rev. 177 (1969) 151.

[7] SAmson, J. A. R., Phil. Trans. R. Soc. A 268 (1970) 141.

[8] Morgenstern, R., Niehaus, A. and Ruf, M. W., Chem. Phys. Lett. 4 (1970) 635

[9] Carlson, T. A. and Jonas, A. E., J. Chem. Phys. 55 (1971) 4913.

[10] Niemaus, A. and Ruf, M. W., Z. Phys. 252 (1972) 84.

[11] Mitchell, P. and Codling, K., Phys. Lett. 38A (1972) 31.

[12] Lynch, M. J., Gardner, A. B. and Codling, K., Phys. Lett. 40A (1972) 349

[13] Lynch, M. J., Codling, K. and Gardner, A. B., Phys. Lett. 43A (1973) 213.

[14] Wulleumier, F. and Krause, M. O., Phys, Rev. A 10 (1974) 242.

[15] Watson, W. S. and Stewart, D. T., J. Phys. B 7 (1974) L466.

[16] Houlgate, R. G., West, J. B., Codling, K. and Marr, G. V., J. Phys. B 7 (1974) L470; J. Electron Spectrosc. 9 (1976) 205.
[17] VAN DeR wiel, M. J. and Brion, C. E., J. Electron Spectrosc. $1(1973) 439$

[18] Branton, G. R. and Brion, C. E., J. Electron Spectrosc. 3 (1974) 123.

[19] Dehmer, J. L., Chupka, W. A., Berkowitz, J. and Jivery, W. T. Phys Rev. A 12 (1975) 1966

[20] Torop, L., Morton, J. and West, J. B., J. Phys. B 9 (1976) 2035.

[21] Codling, K., Houlgate, R. G., West, J. B. and WoodrufF, P. R., J. Phys. B 9 (1976) L83.

[22] Hancock, W. H. and Samson, J. A. R., J. Electron Spectrosc. 9 (1976) 211

[23] Manson, S. T., J. Electron Spectrose. 1 (1972) 413.

[24] Kennedy, D. J. and Manson, S. T., Phys. Rev. A 5 (1972) 227.

[25] Amusia, M. Ya., Cherepkov, N. A. and Chernysheva, L. V., Phys. Lett. 40A (1972) 15.

[26] Bethe, H., in Handbuch der Physik, edited by H. Geiger and K. Scheele (Springer, 1933) Vol. 24, p. 273.

[27] Sommerfeld, A. and Schur, G., Ann. Phys. 4 (1930) 409 SCHUR, G., Ann. Physik 4 (1930) 433.

[28] OpPenheimer, J. R., unpublished; referred to in E. C. Watson and J. A. van den Akker, Proc. R. Soc. (London) A 126 (1929) 138

[29] Yang, C. N., Phys. Rev. 74 (1948) 764

[30] Cooper, J. and Zare, R. N., J. Chem. Phys. 48 (1968) 942; and in Lectures in Theoretical Physics, Eds. S. Geltman, K. Mahanthappa and N. Brittin (Gordon and Breach, New York) 1969, Vol. 1Ic, p. 317 
[31] Dill, D. and FANo, U., Phys. Rev. Lett. 29 (1972) 1203.

[32] Fano, U. and Dill, D., Phys. Rev, A 6 (1972) 185.

[33] JACOBS, V. L., J. Phys. B 5 (1972) 2257.

[34] Dill, D., Phys. Rev. A 7 (1973) 1976.

[35] Walker, T. E. H. and Waber, J. T., J. Phys. B 6 (1973) 1165.

[36] Dill, D., Starace, A. F. and Manson, S. T., Phys. Rev. A 11 (1975) 1596.

[37] Tully, J. C., Berry, R. S. and Dalton, B. J., Phys. Rev. 176 (1968) 95.

[38] Buckingham, A. D., OrR, B. J. and Sichel, J. M., Phil. Trans. R. Soc. London A 268 (1970) 147.

[39] Dill, D., Phvs. Rev. A 6 (1972) 160

[40] Dehmer, J. L. and Dill, D., J. Chem. Phys. 65 (1976) 5327.

[41] Druger, S., J. Chem. Phys. 64 (1976) 987.

[42] Fano, U. and Cooper, J. W., Rev. Mod. Phys, 40 (1968) 441.

[43] Dill, D., in Photoionization and Other Probes of Many-Electron Interactions, F. J. Wuilleumier, Ed. (Plenum, N.Y.) 1976 , p. 387.

[44] Starace, A. F., Manson, S. T. and Kennedy, D. J., Phys. Rev. A 9 (1974) 2453.

[45] Manson, S. T., Kennedy, D. J., Starace, A. F. and Dill, D., Planet. Space Sci. 22 (1974) 1535

[46] Dill, D., Manson, S. T. and Starace, A. F., Phys. Rev. Lett. 32 (1974) 971.

[47] Starace, A. F., Rast, R. H. and Manson, S. T., Phys. Rev. Lett. 38 (1977) 1522.

[48] Manson, S. T., Starace, A. F. and Dill, D., Bull. Am. Phys. Soc. 19 (1974) 1203.

[49] Manson, S. T., Starace, A. F. and Dill, D., Ninth International Conference on the Physics of Electronic and Atomic Collisions : Abstracts of Papers (U. of Washington, Seattle) 1975 , p. 569

[50] Manson, S. T., Msezane, A. and Starace, A. F., Fifth International Conference on Atomic Physics (Berkeley, California) 1976 , p. 427.

[51] Dehmer, J. L. and Dill, D., Phys. Rev. Leti. 37 (1976) 1049.

[52] Samson, J. A. R. and Hancock, W. H., Phys. Lett. A 61 (1977) 380

[53] Dehmer, J. L. and Dehmer, P. M, J. Chem. Phys. 67 (1977) 1782.

[54] Walker, T. E. H., Berkowitz, J., Dehmer, J. L. and Waber, J. T., Phys. Rev. Lett. 31 (1973) 678.

[55] Walker, T. E. H. and Waber, J. T., J. Phys. B 7 (1974) 674.

[56] Ron, A., Pratt, R. H. and Tseng, H. K., Chem. Phys. Lett. 47 (1977) 377

[57] Tseng, H. K., Pratt, R. H., Yu, S. and Ron, A., Phys. Rev. A, to be published.
[58] Samson, J. A. R. and Gardner, J. L., J. Opt. Soc. Am. 62 (1972) 856

[59] Dill, D., Argonne National Laboratory Radiological and Environmental Research Division Annual Report, July 1972-June 1973, ANL-8060, Part I, p. 190.

[60] LeE, C. M., Phys. Rev. A 10 (1974) 1598.

[61] Samson, J. A. R. and Gardner, J. L., Phys. Rev. Lett. 31 (1973) 1327.

[62] Carlson, T. A., McGuire, G. E., Jonas, A. E., Cheng, K. L., Anderson, C. P., LU, C. C. and Pullen, B. P., in Electron Spectroscopy, ed. D. A. Shirley (North-Holland, Amsterdam) 1972, p. 207

[63] Carlson, T. A., Chem. Phys. Lett. 9 (1971) 23.

[64] Dehmer, P. M. and Chupka, W. A., J. Chem. Phys. 62 (1975) 4525 .

[65] Duzy, C. and Berry, R. S., J. Chem. Phys. 64 (1976) 2421.

[66] Niehaus, A. and Ruf, N. W., Chem. Phys. Lett. 11 (1971) 55.

[67] Ritchie, A. B., Phys. Rev. Lett., to be published.

[68] Rabalais, J. W., Debies, T. P., Berkosky, J. L., Huang, J.-T. J. and Ellision, F. O., J. Chem. Phys. 61 (1974) 529.

[69] Iwata, S. and Nagakura, S., Mol. Phys. 27 (1974) 425.

[70] Dehmer, J. L. and Dill, D., J. Chem. Phys., to be published.

[71] Dill, D., Siegel, J. and Dehmer, J. L., J. Chem. Phys. 65 (1976) 3158

[72] Davenport, J. W., Phys. Rev. Lett. 36 (1976) 945.

[73] Dill, D. and DeHMer, J. L., J. Chem. Phys. 61 (1974) 692.

[74] Itikawa, Y., Chem. Phys., to be published.

[75] Itikawa, Y., Chem. Phys., to be published.

[76] WIGHT, G. R., BRION, C. E. and VAN DER WIEL, M. J., J. Electron Spectrosc. 1 (1973) 457.

[77] Robinson, A. L., Science 196 (1977) 1306.

[78] ZARE, R. N., Mol. Photochem. 4 (1972) 1.

[79] YANG, S.-C. and Bersohn, R., J. Chem. Phys. 61 (1974) 4400

[80] Ormerod, R. C., Anderson, W. R. and Rose, T. L., J. Chem. Phys. 62 (1975) 127.

[81] Ling, J. H. and Wilson, K. R., J. Chem. Phys. 65 (1976) 881.

[82] Ormerod, R. C., Powers, T. R. and Rose, T. L., J. Chem. Phys 60 (1974) 5109.

[83] Stone, E. J., Lawrence, G. M. and Fairchild, C. E., J. Chem. Phys. 65 (1976) 5083.

[84] Sharp, T. E., At. Data 2 (1971) 119.

[85] BaCKX, C., Wight, G. R. and VAN DER Wiel, M. J., J. Phys. B 9 (1976) 315 and references therein.

[86] Gardner, J. L. and Samson, J. A. R., Phys. Rev. A 12 (1975) 1404.

[87] Wood, R. M., Edwards, A. K. and Steuer, M. F., Phys. Rev. A 15 (1977) 1433. 